\title{
8. Umgang mit aggressivem Verhalten
}

\section{Cornelia Klinger}

Die ärztliche Behandlung von psychisch kranken Menschen beinhaltet im stationären Alltag auch die Auseinandersetzung mit einem Thema, das bei den meisten Menschen zunächst berechtigte Angst hervorruft: der Umgang mit aggressivem Verhalten. Dieses Problem ist deshalb so besonders, weil die eigene emotionale oder sogar physische Betroffenheit bei aggressiven Auseinandersetzungen mit Patienten das sachliche und rasche ärztliche Handeln nicht beeinträchtigen darf.

Gerade für Anfänger auf dem Gebiet der Psychiatrie ist dies eine große Belastung und wirkt oftmals erschreckend. Eine Gewöhnung an aggressive Verhaltensweisen oder sogar eine echte Routine im Umgang damit gibt es auch nach mehreren Berufsjahren kaum.

Jedoch gibt es praktische Empfehlungen, die auf dem Boden von wissenschaftlichen Erkenntnissen, ethischen Überlegungen und den gesetzlichen Bestimmungen entstanden sind und die es ermöglichen, Sicherheit für Patienten und Beschäftigte der Klinik herzustellen und dabei die Menschenwürde aller Beteiligten zu wahren. Diese sind in der S2-Leitlinie der Deutschen Gesellschaft für Psychiatrie, Psychotherapie und Nervenheilkunde „Therapeutische Maßnahmen bei aggressivem Verhalten“ aus dem Jahre 2010 zusammengefasst, auf die in der folgenden Darstellung immer wieder Bezug genommen wird.

Den therapeutischen Maßnahmen bei aggressivem Verhalten ist voranzustellen, dass der Prävention solcher Ereignisse in der psychiatrischen Arbeit ein hoher Stellenwert einzuräumen ist. Aggression findet in Beziehungen statt. Die Beziehung zu den Patienten wird in der Klinik von allen Berufsgruppen gestaltet (Leitlinie S. 29 [im Folgenden LL]). Respekt und Würde sollten stets den Umgang mit den Patienten prägen. Es muss durch alle an der Behandlung Beteiligten frühzeitige und ausreichende Informationen sowie nachgehende Erklärungen über alle stattgefundenen Behandlungsmaßnahmen gegenüber dem Patienten geben, die zudem seinem aktuellen Verständnisvermögen angepasst sind. Neben der Beziehungsgestaltung tragen Setting und Milieugestaltung sowie klare und transparente Strukturen auf der Station zur Reduktion aggressiver Vorfälle bei (LL, 27). 


\section{Erkennung aggressiven Verhaltens}

Der aussagekräftigste Prädiktor für Aggression ist eine Vorgeschichte mit aggressivem Verhalten (LL, 18). Die Anamnese des Patienten sollte unter diesem Aspekt studiert bzw. das Pflegepersonal, das den Patienten eventuell aus früheren Krankheitsphasen kennt, gezielt danach befragt werden.

Bei der Beurteilung, ob ein Patient aggressives Verhalten zeigen könnte, sind folgende psychische Verhaltenstendenzen zu beachten:

- feindselige Attributionen

- Tendenz zur höheren Gewichtung negativer Informationen

- rigide kognitive Schemata

- eingeschränkte soziale Fertigkeiten

- eingeschränkte Fähigkeit zur Empathie und Perspektivübernahme

- eingeschränkte Impulskontrolle

Frühwarnzeichen, die auf eine nahende Eskalation hinweisen, sind:

- drohende Körperhaltung und Gestik

- geringe Körperdistanz zwischen Mitarbeitern und Patienten

- verbale Bedrohungen und Beschimpfungen

- psychomotorische Erregung und Anspannung

- Sachbeschädigungen

- gesteigerte Tonhöhe und Lautstärke

Wenn Gewalttätigkeit vermutet werden muss, ist es zunächst erforderlich, den Patienten intensiv zu beobachten und zu begleiten. Diese vom Arzt anzuordnende und vom Pflegepersonal durchzuführende Maßnahme beinhaltet regelmäßige Kontakte im festzulegenden Zeitabstand (z.B. halbstündlich oder viertelstündlich) bis hin zur Einzelbetreuung. Die Kontakte dienen neben der frühzeitigen Wahrnehmung von eskalierendem Verhalten auch der therapeutischen Einflussnahme.

\section{Interventionen}

Eine zwangsweise Behandlung eines Patienten kann sowohl der Gefahrenabwehr als auch der Besserung des Krankheitszustandes selbst dienen. Die Durchsetzung einer Zwangsbehandlung ist ein massiver Eingriff in die Autonomie eines Menschen und bedarf äußerst sorgfältiger und wiederholter Prüfungen. Sie ist nur legitimiert, wenn die freie Willensbildung durch die psychische Erkrankung partiell oder vollständig aufgehoben ist und die Fremdgefährdung eine kausale Folge der Erkrankung darstellt (LL, 49). Ziel muss es sein, die am wenigsten in das Selbstbestimmungsrecht des Patienten eingreifende Maßnahme zu wählen.

Im Stationsalltag empfiehlt es sich, im Behandlungsteam abzusprechen, wer eine vom Patienten positiv bewertete Beziehung aufbauen konnte und daher besonders geeignet ist und sich selbst in der Lage fühlt, einen kommunikativen $\mathrm{Zu}$ - 
gang zu finden und deeskalierend wirken zu können. Dies muss nicht in jedem Fall einer drohenden Eskalation der behandelnde Arzt sein!

Spätestens an dieser Stelle muss überprüft werden, ob eine Durchsuchung auf Waffen oder gefährliche Gegenstände bereits erfolgt ist oder ob solche in Reichweite des Patienten gelangt sein könnten.

\section{Verbale Deeskalation}

Die verbale Deeskalation einer potenziell gewalttätigen Situation hat zum Ziel, die emotionale Anspannung des Patienten zu reduzieren. Dies gelingt am besten, wenn der Arzt deutlich machen kann, dass er ein echtes Interesse an der Sichtweise des Gegenübers hat und dass die mitgeteilten ärger- oder angstauslösenden Probleme ernst genommen werden. Hierbei sollten Ich-Botschaften verwendet werden (Bsp.: „Ich erlebe es so ...“ und nicht: „Das ist so!“).

Während eines verbalen Deeskalationsversuchs muss immer eine Risikoabschätzung erfolgen, inwieweit die angespannte Situation überhaupt noch ohne Einsatz physischer Mittel zu bewältigen ist. In der klinischen Praxis hat es sich bewährt, diese Risikoabwägung möglichst zusammen mit den Pflegekräften vor Ort vorzunehmen.

\section{Fixierung}

Wenn alle anderen Deeskalationsversuche erfolglos blieben, sind freiheitseinschränkende Zwangsmaßnahmen das Mittel der letzten Wahl, um Gefahren für den Patienten selbst und andere Menschen abzuwenden. Nur der Arzt darf nach eigener Untersuchung des Patienten und Urteilsbildung eine Fixierung anordnen. Die Gründe für die Fixierung und die vorausgegangenen Deeskalationsversuche müssen schriftlich dokumentiert werden.

Eine Fixierung erfolgt mittels Gurtsystemen am Bett, um die Bewegungsmöglichkeit des Patienten weitgehend einzuschränken. Dabei sollte mindestens eine 4-Punkt-Fixierung erfolgen, also an beiden Armen und beiden Beinen, da sonst erhebliche Verletzungsgefahren für den Patienten drohen. Bei höchstagitierten Patienten können auch eine 5-Punkt-Fixierung (zusätzlicher Bauch- oder Schultergurt) oder sogar weitere Fixierungspunkte erforderlich werden.

Vor der Fixierung selbst sollte vom Arzt sichergestellt werden, dass eine ausreichende Anzahl von Mitarbeitern anwesend ist, um so sicher und so rasch wie möglich handeln zu können.

Trotz der schwierigen Situation, in der sich alle Beteiligten befinden, sollte die Überwältigung des Patienten von Bemühen um eine möglichst würdevolle Behandlung geprägt sein. Hierzu zählt, dass Patienten nicht vollständig entkleidet werden sollten.

Bei einer notwendigen Fixierung von Patientinnen muss beachtet werden, dass es wegen der hohen Prävalenz von posttraumatischen Störungen unter psychisch kranken Frauen zu einer Retraumatisierung kommen kann. Hierbei muss sich 
besonders um Alternativen, z.B. kontinuierliche Überwachung anstelle von Fixierung, bemüht werden.

Der fixierte Patient wird für die Dauer der Maßnahme in Einzelbetreuung durch eine Pflegekraft kontinuierlich überwacht und begleitet.

Eine Aufklärung des Patienten über die Fixierung und die beabsichtigte Dauer ist erforderlich. Die Dauer der Fixierung ist so kurz wie möglich zu halten, die Indikation zur Fixierung ist durch eine ärztliche Untersuchung des Patienten engmaschig zu überprüfen.

\section{Pharmakologische Interventionen}

Wenn das aggressive Verhalten bei einer psychiatrischen Erkrankung mit einem psychomotorischen Erregungszustand einhergeht, ergibt sich die Indikation für eine pharmakologische Behandlung. Dies stellt einen psychiatrischen Notfall dar und erfordert unmittelbares Handeln.

Auch hier ist vor der Entscheidung zur zwangsweisen Applikation eines Medikaments der Versuch zu unternehmen, den Patienten zur Kooperation zu bewegen. Wenn das Einverständnis des Patienten erreicht werden kann, ist in der Regel die orale Medikation vorzuziehen. Wenn der Patient vor der Erkrankung Äußerungen zur Behandlung in einem solchen Fall getätigt hat (z.B. im Rahmen einer Behandlungsvereinbarung), ist dies zu berücksichtigen.

Die medikamentöse Zwangsbehandlung erfordert eine klare medizinische Indikation und erfolgt auf rechtlicher Grundlage (s. Kap. III.4).

Das Ziel einer pharmakologischen Intervention ist die Sedierung. Hinsichtlich der Substanzwahl besteht die Schwierigkeit, dass sich die verfügbaren Studienergebnisse auf Patientenbehandlungen beziehen, die den Einschlusskriterien der Studien entsprochen haben. Im klinischen Alltag bestehen häufig andere Bedingungen (z.B. begleitende Intoxikationen, kein Informed Consent möglich), sodass sich die in der Leitlinie empfohlenen Substanzen zusätzlich auf klinische Erfahrungen stützen (LL, 65).

Die Substanzwahl im Einzelnen bleibt den erprobten und festgelegten Standards der jeweiligen Klinik überlassen. Neue Erkenntnisse über unerwünschte Arzneimittelwirkungen müssen dabei dringend beachtet werden. Dies gilt auch für Substanzen, die bereits über Jahrzehnte eingesetzt wurden. Zum Beispiel haben neue Daten über QTc-Zeit-Verlängerungen bei Haloperidol $2010 \mathrm{zu}$ einer Einschränkung der Zulassung dieses Medikaments geführt. Die parenterale Gabe wird nur noch intramuskulär empfohlen. Eine intravenöse Applikation bedarf eines besonderen kardialen Monitorings.

Wenn aggressive Erregungszustände mit Intoxikationen von Alkohol, Drogen oder unbekannten Substanzen einhergehen, ist die Indikation zum Einsatz von sedierenden Medikamenten außerhalb intensivmedizinischer Behandlungsmöglichkeiten wegen der Gefahr der Atemdepression kritisch zu stellen. 


\section{Nachbesprechung von aggressivem Verhalten mit dem Patienten}

Nachbesprechungen von aggressivem Verhalten und Zwangsmaßnahmen bieten die Möglichkeit, systematisch die Erfahrungen und Sichtweisen der Patienten in der Behandlungsplanung zu berücksichtigen, präventive Maßnahmen zu vereinbaren, die Patienten emotional entlasten und das möglicherweise traumatisierende Ereignis zu verarbeiten (LL, 82).

Ein rückblickendes Gespräch über die abgelaufene Zwangsmaßnahme sollte daher in Abhängigkeit vom Befinden des Patienten in jedem Fall durchgeführt werden. Gesprächsteilnehmer sind der Patient, der die Zwangsmaßnahme anordnende Arzt sowie eine pflegerische Bezugsperson. Inhaltlich sollten die Darstellung der (häufig unterschiedlichen) Sichtweisen von Patient und Mitarbeitern im Vordergrund stehen sowie das Gespräch für Absprachen zur Vermeidung künftiger Eskalationen genutzt werden. Das Gespräch wird in der Krankenakte dokumentiert.

Von aggressivem Verhalten betroffenen Mitpatienten müssen zeitnah Einzelgespräche zur Bewältigung des Erlebten angeboten werden.

\section{Betreuung der Mitarbeiter, die von Patientenübergriffen betroffen waren}

Wenn Mitarbeiter von einem aggressiven Vorfall betroffen, d.h. physisch oder psychisch Gewalt ausgesetzt sind, muss nach dem Vorfall unmittelbar eine kollegiale Unterstützung erfolgen. Als weitergehende Nachsorge, falls gewünscht, sollte ein in der Klinik etabliertes Nachsorgeteam Gesprächskontakte anbieten.

\section{Fazit}

Abschließend ist zu sagen, dass jede aggressive Auseinandersetzung mit einem Patienten ein Erlebnis im ärztlichen Tun ist, das jedem nahe geht und wohl niemandem gleichgültig ist. Diese Ergriffenheit sollte genutzt werden, um immer wieder Vorgehensweisen, Kommunikationsstrategien und Handlungsimpulse in sich selbst zu erforschen. Es ist überdies sinnvoll für die Bewältigung solcher schwierigen Erlebnisse, die eigenen Erfahrungen im Behandlungsteam zu besprechen, zu reflektieren und gemeinsam die emotionale Belastung zu tragen.

\section{Literaturempfehlung}

S2 Praxisleitlinie in Psychiatrie und Psychotherapie (Hrsg. Deutsche Gesellschaft für Psychiatrie, Psychotherapie und Nervenheilkunde [DGPPN]) (2010) Therapeutische Maßnahmen bei aggressivem Verhalten in der Psychiatrie und Psychotherapie (erstellt von einer Leitlinienprojektgruppe unter Federführung von Tilman Steinert), Steinkopff Verlag Frankfurt/Main 